\title{
Mortalità nei pazienti emodializzati: predirla e (cercare di) ridurla
}

\author{
Andrea Cavalli, Giuseppe Pontoriero \\ S.C. Nefrologia e Dialisi, Ospedale "Alessandro Manzoni", Lecco
}

\begin{abstract}
Mortality in hemodialysis patients: predict and (trying to) reduce it
Mortality risk scores for hemodialysis patients have been developed also in the past, but with low clinical applicability. Floege has recently developed a score in 11.500 hemodialysis incident European patients, able to predict mortality risk after 1 and 2 years. The principal determinants of the score were: older age, low body mass index, history of cancer, high levels of C-reactive protein and low levels of albumin. The relevance of the score is due to its high applicability also to an external cohort of hemodialysis patients (the DOPPS study patients). The increased incidence of cancers in hemodialysis patients represents another important situation, in which we need to improve our everyday clinical practice, especially for cancers frequent in dialysis patients (kidney/renal pelvis and bladder), in order to apply adequate screening procedures.
\end{abstract}

Keywords: Hemodialysis, Hyperkalemia, Mortality, Neoplasia, Risk score, Vascular calcification

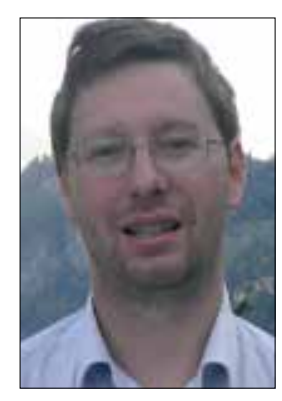

Andrea Cavalli
Sappiamo tutti molto bene come i pazienti affetti da insufficienza renale cronica (IRC) e, in particolare, quelli in trattamento dialitico presentino un'elevata mortalità, legata specialmente a cause cardiovascolari e infettive, quantificabile intorno ai 19.2 soggetti ogni 100 persone per anno (superiore rispetto a quella associata a diversi tipi di neoplasie), rispetto a 1.2 soggetti ogni 100 persone per anno riscontrabili nella popolazione generale europea (1). La capacità di identificare quei pazienti a più elevato rischio di morte sarebbe utile per numerosi motivi: riconoscere i fattori su cui poter intervenire per cercare di migliorarne la prognosi, avere dei parametri ai quali fare riferimento quando si propone la terapia dialitica o il trapianto renale, uniformare la popolazione arruolata in studi clinici e poter eseguire valutazioni di economia sanitaria.

In passato, sono stati sviluppati degli score di rischio per la valutazione della mortalità nel dializzato (gli indici di Wright-

Accepted: February 14, 2016

Published online: February 26, 2016

Indirizzo per la corrispondenza:

Prof. Giuseppe Pontoriero

S.C. Nefrologia e Dialisi

Ospedale "Alessandro Manzoni"

Via dell'Eremo 9/11

23900 Lecco

g.pontoriero@ospedale.lecco.it
Khan, Foley o Couchoud) (2-4), che, però, non si sono dimostrati applicabili a popolazioni più ampie rispetto a quella studiata né statisticamente affidabili.

In quest'ambito, sono stati recentemente pubblicati i risultati di uno studio multicentrico europeo, volto a sviluppare degli score di rischio di morte, a 1 e a 2 anni dall'inizio dell'emodialisi, che potessero essere anche validati esternamente, in altre popolazioni di dializzati (5). Oltre 11.500 adulti incidenti in emodialisi (senza storia di pregresso trapianto renale) presso più di 300 centri Fresenius di 14 stati europei sono stati arruolati tra il Gennaio del 2007 e il Dicembre del 2009 nella coorte denominata AROii ("second Analyzing Data, Recognizing Excellence and Optimizing Outcomes") (5).

I punteggi di rischio della coorte AROii sono stati derivati con un approccio simile a quello usato per il famoso studio Framingham (6) e il potere predittivo di ogni fattore di rischio è stato calcolato per mezzo di un modello di regressione di Cox. I punteggi totali rilevati per ogni paziente sono stati, poi, suddivisi in terzili di rischio, osservando come 8-9 punti fossero il cut off che permetteva di distinguere pazienti a basso e ad alto rischio.

Durante il primo e il secondo anno di follow up sono stati riportati rispettivamente 1060 (10.9\%) e 654 (9.4\%) decessi, determinando un tasso di mortalità a 1 e a 2 anni rispettivamente di 13 e 11.2 ogni 100 soggetti.

Nell'analisi presentata, emergeva come un'età avanzata (maggiore di 70 anni, ma, soprattutto, maggiore di 80 anni), un basso indice di massa corporea (inferiore a 18.5) e un' anamnesi positiva per malattia cardiovascolare o neoplasia fossero associati a un'aumentata mortalità a 1 e a 2 anni. L'utilizzo di un catetere venoso centrale e un basso flusso 
ematico (inferiore a $267 \mathrm{~mL} / \mathrm{min}$ ) dell'accesso vascolare si sono dimostrati i parametri di dialisi associati a un outcome peggiore. In termini di parametri di laboratorio, tale evidenza è, invece, emersa per bassi valori di emoglobina (minore di $10 \mathrm{~g} / \mathrm{dL}$ ), elevati livelli di indici infiammatori (proteina $\mathrm{C}$ reattiva maggiore di $18.2 \mathrm{mg} / \mathrm{L}$ e ferritina superiore a $500 \mathrm{mcg} / \mathrm{L}$ ), ipoalbuminemia (inferiore a $3.5 \mathrm{~g} / \mathrm{dL}$ ) e bassi valori di creatininemia pre-dialisi (al di sotto di $431 \mathrm{mmol} / \mathrm{L} / 4.87 \mathrm{mg} / \mathrm{dL}$ ), che riflettono, evidentemente, uno stato di ridotta alimentazione e di progressiva perdita di massa muscolare (Tab. I).

La presenza di una nefropatia diabetica o tubulointerstiziale come nefropatia primitiva e il fumo, pregresso o attuale, esponevano i soggetti a una minore sopravvivenza a 2 anni (ma non a 1 anno), mentre l'ipercalcemia era rilevante solo nella prognosi a 1 anno (Tab. I).

I risultati di questo studio non cambiavano anche qualora si considerassero i primi 90 giorni di follow up del paziente, dopo la prima seduta emodialitica.

Nel momento in cui i rischi relativi sono stati convertiti in punteggi di rischio, l'età avanzata ne determinava il maggior punteggio (4 punti tra 70 e 79 anni, 6 se più di 80 anni) e un'anamnesi positiva per neoplasia incideva maggiormente rispetto a precedenti eventi cardiovascolari (rispettivamente 4 e 3 punti rispetto a 2 e 1 nella prognosi a 1 e 2 anni), mentre, tra i parametri di laboratorio, quello più significativo era rappresentato da elevati livelli di proteina $C$ reattiva (determinante 5 e 4 punti a 1 e a 2 anni se superiore a $18.2 \mathrm{mg} / \mathrm{L}$ ), seguito dall'ipoalbuminemia ( 3 e 2 punti a 1 e a 2 anni se inferiore a $3.5 \mathrm{~g} / \mathrm{dL}$ ), da bassi valori di creatininemia e da emoglobina inferiore a $10 \mathrm{~g} / \mathrm{dL}$. Parametri che si dimostravano "protettivi", ovvero che determinavano una riduzione dello score, erano rappresentati da giovane età (-5 punti se inferiore a 39 anni e -2 se tra i 40 e i 49 anni), emoglobina superiore a $12 \mathrm{~g} / \mathrm{dL}$, ferritina inferiore a $500 \mathrm{mcg} / \mathrm{L}$, indice di massa corporea superiore o uguale a 30 , flusso ematico a livello dell'accesso vascolare di almeno $267 \mathrm{~mL} / \mathrm{min}$ (tutti parametri associati a -1 punto sul punteggio totale).

Fissando intorno a 8-9 punti il livello per distinguere i pazienti a basso e ad alto rischio, il punteggio si dimostrava altamente sensibile nel predire il rischio di morte a 1 e a 2 anni (sensibilità rispettivamente pari all'81.5\% e al $70.7 \%$ ), anche se decisamente meno specifico (specificità rispetti- vamente pari al $56.4 \%$ e al $66 \%$ ). La suddivisione in terzili di rischio permetteva di distinguere efficacemente gruppi di pazienti a basso, medio e alto rischio di morte ( $P$-value $<0.001)$.

La forza dei risultati del lavoro di Floege et al (5) sta nel fatto che la predittività dei punteggi di rischio a 1 e a 2 anni rimaneva valida anche quando veniva applicata ad altre popolazioni di dializzati, come a quella della terza fase dello studio DOPPS (scelta per contemporaneità di decorso e confrontabilità di dati, anche se costituita da pazienti prevalenti e non incidenti), mantenendo anche quell'aumento di mortalità osservato, parallelamente al crescere dei terzili di punteggio.

L'applicazione dello score di AROii permetteva, inoltre, di aumentare il potere predittivo degli score precedentemente sviluppati, come quello di Wright-Khan (2).

Gli Autori hanno, pertanto, presentato un nuovo modello predittivo basato sull'utilizzo di semplici dati anamnestici, dialitici e di laboratorio, dotato di sensibilità statistica rispetto a precedenti modelli di rischio ben più complicati. La capacità di predire la prognosi dei pazienti si è dimostrata applicabile a pazienti incidenti, sin dal primo giorno di emodialisi, permettendone, quindi, l'estensione anche a quei soggetti in cui si dubiti possa esserci un reale vantaggio nell'iniziare il trattamento dialitico. L'applicabilità dello score allo studio DOPPS permette di estenderne la validità anche a pazienti prevalenti, oltre a costituire un elemento di novità rispetto ai precedenti modelli di rischio.

Inoltre, la semplicità del modello ne permette anche la facile implementazione nelle tecnologie odierne (come un'applicazione del cellulare), vantaggio che non sarebbe possibile con modelli più sofisticati, dove la maggiore complessità sicuramente penalizzerebbe un'eventuale (lieve) migliore accuratezza prognostica.

Lo score prognostico di AROii non è, però, esente da limitazioni (Tab. II): essendo stato elaborato in soggetti emodializzati non è generalizzabile a pazienti in dialisi peritoneale, non ha considerato la severità delle comorbidità (al fine di non rendere troppo complicato il modello e per non introdurre soggettività nella valutazione) e non ha utilizzato altre importanti variabili (essere un "late referral", la dipendenza nei trasporti e la qualità della vita, per esempio), che avrebbero potuto migliorare il potere predittivo del punteggio.

In aggiunta, occorre ricordare come gli score di rischio

TABELLA I - Parametri considerati nello score di aroii

\begin{tabular}{ll}
\hline Non modificabili & Modificabili \\
\hline Età & Fumo* \\
Anamnesi positiva per malattie cardiovascolari & Indice di massa corporea (BMI) \\
Anamnesi positiva per neoplasie & Accesso vascolare per emodialisi (tipologia e flusso ematico) \\
Eziologia della malattia renale cronica* & Parametri di laboratorio (emoglobina, ferritinemia, proteina C reattiva, al- \\
& buminemia, creatininemia e calcemia^) \\
\hline
\end{tabular}

* Non rilevante nella prognosi a 1 anno.

$\wedge$ Non rilevante nella prognosi a 2 anni. 
TABELLA II - Limiti dello score prognostico di AROii

- Elaborato solo in soggetti emodializzati
- Esclusione dei soggetti con precedente trapianto renale
- Pazienti appartenenti unicamente a centri dialisi Fresenius
- Non considerata la severità delle comorbidità
- Non valutata la prognosi a più lungo termine ( 3 e 5 anni)
- Non valutate variabili come la qualità della vita, la dipendenza
nella mobilizzazione o l'essere "late referral"
- Specificità dello score nettamente inferiore rispetto alla sensibilità
- Esprime solo associazioni con una prognosi e non un rapporto di
causalità

TABELLA III - Messaggi derivanti dallo studio di Butler

- Incidenza aumentata di neoplasie negli emodializzati rispetto alla popolazione generale

- Neoplasie più frequenti nei soggetti maschi di più di 65 anni, senza nefropatia diabetica

- Incremento delle neoplasie particolarmente significativo a livello di reni/pelvi renale e vescica

- Opportunità di instaurare delle procedure di screening (ecografia dei reni nativi e delle vie urinarie, per esempio), almeno in popolazioni selezionate

- Indagare approfonditamente tutti gli episodi di ematuria/perdite ematiche a livello delle vie urinarie

possano solo descrivere delle associazioni con una prognosi, aiutando, quindi, a generare delle ipotesi, e non permettano, invece, di stabilire dei rapporti di causalità, che deriverebbero solo da studi clinici randomizzati, controllati e in doppio cieco.

Vogliamo anche aggiungere come sia sempre necessario valutare il paziente nella sua globalità e non limitandosi solo all'utilizzo di punteggi, che potrebbero rischiare di farci perdere una visione più umana e olistica dei soggetti, che potrebbe, invece, derivare dal lungo follow up ambulatoriale pre-dialitico che spesso ha preceduto l'ingresso in dialisi. Uno score di questo tipo, migliore comunque dei precedenti, rappresenta pertanto solo uno dei possibili elementi utili per rispondere alla domanda che spesso ci viene posta: "Dottore, quanto mi resta da vivere?", per citare l'editoriale di commento all'articolo di Floege (7).

Abbiamo visto come, nello score di AROii, una pregressa neoplasia rappresenti il dato anamnestico più strettamente associato a una prognosi negativa. Diventa, quindi, fondamentale il precoce riconoscimento di tali patologie, anche nella popolazione di dializzati, al fine di poterne ridurre la prognosi negativa ulteriormente associata. Questa associazione è ancora più significativa nel momento in cui si considerano i dati epidemiologici, che indicano un'aumentata incidenza di tumori nei dializzati (8).

II recente lavoro di Butler et al (9) ha descritto l'incidenza di neoplasie nei pazienti emodializzati, dando, però, attenzione (rispetto ai precedenti studi) anche alla valutazione dell'incidenza in vari sottogruppi di soggetti e di distretti corporei, nel periodo 1996-2009, attraverso l'analisi dei dati provenienti dal registro USRDS (US Renal Data System). Sono stati esclusi dalla valutazione i tumori in situ (eccetto quelli mammari e vescicali) e cutanei (considerando, però, i casi di melanoma), le metastasi e i tumori direttamente responsabili di un'insufficienza renale richiedente il trattamento emodialitico. Nel periodo di osservazione, è stato rilevato un incremento costante dell' incidenza di neoplasie (da 3.923 a 3.860 ogni 100.000 personeanno), in particolare per quanto riguarda quelle a carico dei reni e della pelvi renale. È, invece, diminuita l'incidenza dei tumori a intestino, pancreas e polmoni ed è rimasta costante a livello di prostata, mammella, vescica e linfomi non-Hodgkin (9).

Dopo aver aggiustato i dati in accordo alle caratteristiche basali dei pazienti, l'incidenza cumulativa di tumori a 5 anni si rivelava più alta nei soggetti con più di 65 anni all'inizio della dialisi, maschi, neri, con causa di insufficienza renale diversa dalla nefropatia diabetica (forse perché più facilmente affetti da complicanze cardio-vascolari, anche mortali) e valutati per un possibile trapianto renale (forse perché più accuratamente sottoposti a screening anche per neoplasie occulte) (9).

Le localizzazioni più frequenti sono state quelle di prostata, polmoni/bronchi, colon/retto, mammella, reni/pelvi renale e vescica. Rispetto alla popolazione generale americana, i soggetti dello studio hanno presentato un rischio globale superiore di neoplasie $(+42 \%)$, coinvolgente il $9.48 \%$ dei pazienti nell'arco di 5 anni, e, in particolare, ancora più elevato nel caso di reni/pelvi renale (rischio relativo aumentato di circa 4 volte) e vescica $(+57 \%)(9)$.

Lo studio presentato da Butler et al (9) rappresenta la prima analisi di incidenza cumulativa di neoplasie nella popolazione di emodializzati, permettendo, quindi, di ottenere delle nuove informazioni inerenti il rischio oncologico in questi soggetti. Più ipotesi sono state considerate per spiegare tale risultato: carenze nutrizionali, deficit immunologici secondari all'insufficienza renale terminale, potenziale ruolo dell'eritropoietina o eccesso di radiazioni somministrate per esami strumentali.

Considerando che, attualmente, non ci sono delle Linee Guida per lo screening tumorale nei soggetti dializzati, questi recenti dati devono far aumentare la nostra attenzione almeno nei confronti delle categorie di pazienti più a rischio, nelle quali ricercare possibili segni o sintomi suggestivi o applicare procedure volte a una diagnosi precoce, come, per esempio, la regolare esecuzione di un'ecografia annuale dei reni nativi e delle vie urinarie, visto l'eccesso di neoplasie in tali sedi (Tab. III).

Anche in questo caso, quindi, occorre saper individualizzare la nostra attività clinica quotidiana, cercando di prendere in considerazione per ogni nostro paziente le priorità più importanti, che permettano di dar loro non solo "più anni alla vita", ma, soprattutto, "più vita agli anni". 


\section{Disclosures}

Financial support: No financial support was received for this submission.

Conflict of interest: The authors have no conflict of interest.

\section{Bibliografia}

1. de Jager DJ, Grootendorst DC, Jager KJ, et al. Cardiovascular and noncardiovascular mortality among patients starting dialysis. JAMA 2009;302:1782-9.

2. Wright LF. Survival in patients with end-stage renal disease. Am J Kidney Dis 1991;17:25-8.

3. Foley RN, Parfrey PS, Hefferton D, et al. Advance prediction of early death in patients starting maintenance dialysis. Am J Kidney Dis 1994;23:836-45.

4. Couchoud C, Labeeuw M, Moranne O, et al. A clinical score to predict 6-month prognosis in elderly patients starting dialysis for end-stage renal disease. Nephrol Dial Transplant 2009;24:1553-61.

5. Floege J, Gillespie IA, Kronenberg F, et al. Development and validation of a predictive mortality risk score from a European hemodialysis cohort. Kidney Int 2015;87:996-1008.

6. Sullivan LM, Massaro JM, D'Agostino RB Sr. Presentation of multivariate data for clinical use: The Framingham Study risk score functions. Stat Med 2004;23:1631-60.

7. Steenkamp R, Caskey FJ. 'How long have I got doctor?' The development and validation of a new prognostic model. Kidney Int 2015;87:879-82.

8. Maisonneuve $P$, Agodoa L, Gellert R, et al. Cancer in patients on dialysis for end-stage renal disease: an international collaborative study. Lancet 1999;354(9173):93-9.

9. Butler AM, Olshan AF, Kshirsagar AV, et al. Cancer Incidence Among US Medicare ESRD Patients Receiving Hemodialysis, 1996-2009. Am J Kidney Dis 2015;65(5):763-72. 